\title{
Evaluation of Prescribing Pattern of Antiepileptic Drugs and Assessment of Quality of Life of Epileptic Patients and the Knowledge of Their Care Givers
}

\author{
Akhila A. Lekshmi, A. Anjana, Sajila Mary Emmanuel, V. Sreelekshmi, C. D. Shaji Selvin* \\ Sreekrishna College of Pharmacy \& Research Centre, Parassala, Thiruvananthapuram, Kerala, India .
}

\begin{tabular}{l} 
ARTICLE INFO \\
\hline Article history: \\
Received on: $26 / 06 / 2017$ \\
Accepted on: 14/08/2017 \\
Available online: $30 / 10 / 2017$ \\
\hline Key words: \\
Epilepsy, Quality of life, \\
Anti-epileptics, Prescribing \\
pattern, Knowledge, \\
Counseling.
\end{tabular}

\begin{abstract}
A hospital based prospective study was done at a tertiary care hospital over a period of six month to analyze the prescribing pattern of antiepileptic drugs, assess the knowledge of patient's caregivers regarding epilepsy and quality of life of epileptic patients. 105 patients were covered during the study period. It was observed that male population $(63.8 \%)$ was more prevalent to epilepsy than females. Generalized tonic-clonic seizure (52.9\%) were the most common type of epileptic seizure, followed by Complex partial seizure $(22.1 \%)$. Levetiracetam (71.4\%) was the most frequently prescribed, followed by Phenytoin (31.4\%). Poly therapy (59\%) was frequently used. Before counseling, most of the patient's caregivers had a medium level of knowledge regardless of education. Counseling was found to be an effective tool in improving the knowledge of the patient's caregivers. Regarding with the quality of life, the patients were found to have more distress associated with lower energy/fatigue. Seizure worry and social functioning are other domains which have some effects related to quality of life. The study concluded that care of epileptic patients must be taken in to account by considering the various factors influencing the quality of life in these patients.
\end{abstract}

\section{INTRODUCTION}

Epilepsy is a neurological condition distinguished by long-term, frequent and unpredictable happening of seizures (Dahiya et al., 2017). It prevails all over the world with no geographical, racial or social restrictions (Rani et al., 2013). Around 50 million people affected with epilepsy globally (Nimbal et al., 2011). Although, increasing health awareness, the gap between the rate and prevalence of epilepsy in developing and developed countries still exists (Dahiya et al., 2017). In developed countries, the average prevalence rate is $5-6 / 1000$ (Bittencourt et al., 1996; Jallon, 1997; Kuriacose et al., 2014). Comparing with developed countries, the prevalence rate is 2 to 25 times higher in developing countries. Improvement in the care of epileptic patients is unavoidable one due to this high prevalence and also the wrong impressions about this disease. Proper treatment and counseling gain major attention in the

* Corresponding Author

Email: shajimpharm @ gmail.com management of epilepsy (Kuriacose et al., 2014; Long et al., 2000). Special awareness programs to the caregivers of patients helps to reduce the knowledge gaps in the epilepsy management. It also ensures the compliance (Shaju et al., 2014; Elliott et al., 2008). On the other hand, quality of life is an important aspect in the management of epilepsy because it is a chronic and debilitating condition; importantly have multidimensional impact on psychosocial functioning (Rani et al., 2013). With this view, the present study was designed to evaluate the prescribing pattern, quality of life, and the impact of counseling on the knowledge of caregivers of epileptic patients.

\section{METHODS}

After getting necessary approval from institutional ethical committee (R/ADMIN-08/0) a prospective, observational study was conducted for a period of six months from October 2015 to March 2016 in the Department of Neurology, Cosmopolitan Hospitals Pvt. Ltd., a 550 bedded multispecialty hospital located in 
Thiruvananthapuram, Kerala, South India with the object to analyze the prescribing pattern of antiepileptic drugs and quality of life of epileptic patients. The study was also aimed to evaluate the knowledge of patient's care givers regarding epilepsy before and after counseling. Totally, 105 patients meet inclusion criteria were enrolled in the study.

The inclusion criteria were

- Patients admitted in the Neurology Department diagnosed with epileptic seizures and aged between 18 to 70 years.

- Patients with complete medical records and willing to participate in the study.

The exclusion criteria were

- Seizure associated with drug abuse, alcohol withdrawal, chronic kidney and liver disease, hyperthermia.

- Pregnant and breast feeding women.

- Outpatients with seizures.

- $\quad$ Patients with psychiatric problems.

The participants were informed about the study and their consent was received in the prescribed format. The study tool designed was evaluated by experts from the Pharmacy practice Department and from the Neurology Department of Cosmopolitan Hospitals Pvt. Ltd. The tools were reframed based on their valid suggestions. QOLIE-31 questionnaire was used to assess the quality of life of study subjects.

All the information relevant to the study was collected by search from the case records and by conducting interview with the patient and patient's care givers. The Knowledge questionnaire was used to assess the knowledge of patient's care givers. Their responses were marked in the columns provided as "Yes / No / Don't know". The responses were circled according to the number of the respective answer. After the initial assessment of knowledge, the patient and care giver was counseled on various aspects of the disease and treatment with the aid of patient information leaflet. The QOLIE-31 questionnaire was use to collect the data about quality of life of patients. Final interview was done after counseling and the responses were recorded.

The appropriate scores were given to the responses based on the scoring manual of the respective questionnaires. The scores for responses to knowledge questionnaire were given from 0 to 15 . The scoring for QOLIE-31 is given under 7 sections including seizure worry, overall quality of life, emotional well-being, energy/fatigue, cognitive, medication effects and social function. The collected data were recorded in Microsoft Excel sheet and workload was entered as numeric code.

\section{RESULTS}

A prospective, observational study was conducted for a period of six months from October 2015 to March 2016 among the in-patients with epilepsy in the Department of Neurology, Cosmopolitan Hospitals Pvt. Ltd., Thiruvananthapuram, Kerala, South India. 105 patients were covered during the study period.

Regarding with the demographic data, the results showed that 67 of 105 study subjects $(63.8 \%)$ were males and remaining 38 candidates $(36.2 \%)$ were females. According to age, the study population was divided in to five groups. 52 patients $(49.5 \%)$ were aged between $61-70$ years, 20 patients $(19 \%)$ were come under 51 60 years age group. 11 patients $(10.4 \%)$ were aged between $41-50$ years. 14 patients $(13.3 \%)$ were belong to $31-40$ years. Only 8 candidates $(7.6 \%)$ come under below 30 years age group. Regarding with the type of epilepsy, 63 candidates $(60 \%)$ were affected with generalized tonic-clonic seizure, followed by 26 candidates $(24.4 \%)$ were affected with complex partial seizure. 16 candidates $(15.2 \%)$ were affected with simple partial seizure. Analysis of sleep pattern revealed that 54 patients $(51.4 \%)$ had normal sleep, 34 patients $(32.3 \%)$ had disturbed sleep and 17 patients $(16.1 \%$ ) experienced with excess sleep (Table 1).

Table 1: Characteristics of study population.

\begin{tabular}{cccc}
\hline & Characteristics & $\begin{array}{c}\text { No. of } \\
\text { candidates }\end{array}$ & Percentage \\
\hline Gender & Males & 67 & 63.8 \\
& Females & 38 & 36.2 \\
\hline Age & Below 30 & 8 & 7.6 \\
& $31-40$ & 14 & 13.3 \\
& $41-50$ & 11 & 10.4 \\
Type of & $51-60$ & 20 & 19 \\
epilepsy & Generalized tonic-clonic & 63 & 49.5 \\
affected & seizure & & 60 \\
& & & \\
& Complex partial seizure & 26 & 24.4 \\
Sleep pattern & Simple partial seizure & 16 & 15.2 \\
\hline & Normal & 54 & 51.4 \\
& Disturbed & 34 & 32.3 \\
& Excess & 17 & 16.1 \\
\hline
\end{tabular}

Evaluation of prescribing pattern of antiepileptic drugs showed that Levetiracetam $(71.4 \%)$ was the most frequently prescribed anti epileptic drug, followed by Phenytoin (31.4\%), Lorazepam (16.2\%), Sodium valproate and Clobazam individually constituted $11.4 \%$ usage. Phenobarbitone (8.6\%), Carbamazepine and Fosphenytoin individually constituted $6.7 \%$ usage and Clonazepam found a place with $3.8 \%$ usage. It was also observed that $59 \%$ were treated with poly-therapy and remaining $41 \%$ were come under mono-therapy approach. Regarding with the route of administration of drugs, 55 patients $(52.4 \%)$ were under oral administration, 22 patients $(21 \%)$ received the drugs by IV route and 28 patients $(26.7 \%)$ received the drugs by both the route. It was found that, majority of the patients $(84.9 \%)$ were given 'twice daily' followed by 'once daily' dosing of $27.4 \%$. 'Thrice daily dosing' was observed in $17 \%$ patients followed by $4.7 \%$ individually constituted 'at once' and 'when required' category.

Regarding with the bystanders of the patients, the results showed that most of them were in the age group of 51-60 years (33 candidates), followed by the age group less than 30 years (23 candidates), 31-40 years (21 candidates) and 20 candidates belong 
to 41-50 years category. 6 candidates were come under 61-70 years and 2 candidates were above 70 years category (Fig. 1). Regarding with the relationship of bystanders with patients, the results showed that majority of the bystanders, $38.1 \%$ (40 candidates) were wives, followed by 22 candidates $(21 \%)$ were children. Husbands and others individually constituted 15 candidates $(30 \%)$. 3 candidates $(2.9 \%)$ were father. Mother and siblings individually constituted 5 candidates $(9.6 \%)$. The results showed that 59 candidates $(56.1 \%)$ were graduates, 19 candidates $(18 \%)$ were studied up to higher secondary level, 15 candidates (14.2\%) were completed high school level, 11 candidates were postgraduates and only one candidate was uneducated. Regarding with the knowledge of caregivers about epilepsy, the results of initial assessment before counseling showed that, majority of them $(80 \%)$ had medium level of knowledge, next to that, $19 \%$ of bystanders had low level and only $1 \%$ had a high level of knowledge about epilepsy. Results of after counseling assessment revealed that $99 \%$ of bystanders had a high level and only $1 \%$ had medium level knowledge. None of the candidates come under the low level knowledge category. It was identified that the mean value of knowledge score before counseling was 6.6 and after counseling, it was 14.2.

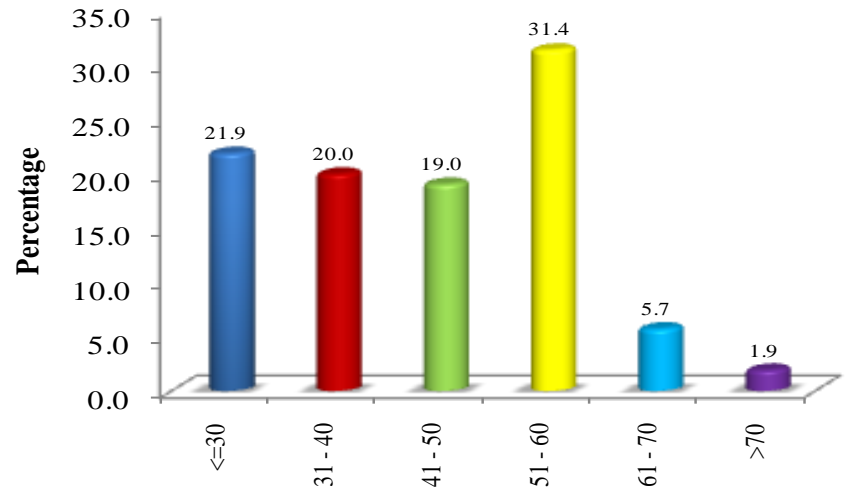

Fig. 1: Age distribution of patient caregivers in percentage.

Regarding with the quality of life of sample population assessed with QOLIE-31 scoring manual, the results showed that about $44.8 \%$ of the patients had an overall quality of life in the score range of $60-80$ and $43 \%$ of the patients had the score of 40 60 . Only $1.9 \%$ of the patients had overall quality of life above the score of 80 . The mean value of quality of life score was 55.9 with a standard deviation of 14.5 (Table 2).

Table 2: Overall quality of life score of study population.

\begin{tabular}{ccc}
\hline Overall quality of life score & No. of patients & Percentage \\
\hline$<40$ & 13 & 12.4 \\
$40-60$ & 43 & 41.0 \\
$60-80$ & 47 & 44.8 \\
$>80$ & 2 & 1.9 \\
Mean \pm SD & $55.9 \pm 14.5$ & \\
\hline
\end{tabular}

Individually, the scoring against various aspects affecting quality of life such as seizure worry, emotional wellbeing, energy/fatigue, cognitive function, medication effects, social functioning were presented in table 3 .

Table 3: Scoring against various aspects affecting quality of life.

\begin{tabular}{ccc}
\hline Aspect & Score & Percentage of patients \\
\hline Seizure worry & $<40$ & 16.2 \\
& $40-60$ & 41.9 \\
& $61-80$ & 29.5 \\
& $>80$ & 12.4 \\
\hline Emotional wellbeing & $<40$ & 20.0 \\
& $40-60$ & 51.4 \\
& $61-80$ & 21.9 \\
& $>80$ & 6.7 \\
\hline Energy fatigue & $<40$ & 36.2 \\
& $40-60$ & 56.2 \\
& $61-80$ & 6.7 \\
& $>80$ & 1.0 \\
\hline Cognitive functioning & $<40$ & 19.0 \\
& $40-60$ & 19.0 \\
& $61-80$ & 36.2 \\
& $>80$ & 25.7 \\
\hline Medication effects & $<40$ & 6.7 \\
& $40-60$ & 12.4 \\
& $61-80$ & 33.3 \\
& $>80$ & 47.6 \\
\hline Social functioning & $<40$ & 16.2 \\
& $40-60$ & 36.2 \\
& $61-80$ & 34.3 \\
& $>80$ & 13.3 \\
\hline
\end{tabular}

\section{DISCUSSION}

The present study highlights the analysis of prescribing pattern of antiepileptic drugs, assessment of knowledge of patient's caregivers, impact of counseling on improvement of knowledge and assessment of quality of life in epileptic patients.

The demographic data indicated that $63.8 \%$ were males and rest were females and was similar to another study from Kerala done by Kuriakose et al., 2014. The dominant age group of the study was $61-70(50.5 \%)$ followed by 51-60 (19\%). It indicates that the majority of study population was elders. This finding was contrast to study conducted in Ethiopia by Rishe et al., 2015 in which the dominant group was young and middle age. Most of the patients were graduates (40\%) followed by $19 \%$ up to high school levels and the data support that patients with epilepsy were educated, and there were no association between the disease and education .This finding was contrast with a study conducted by Kuriakose et al., 2014 in Kerala.

In this study most of the patients were diagnosed with Generalized tonic clonic Seizures (52.9\%), this was similar to that of study conducted by Rishe et al., 2015. 22.1\% of the patients had Complex partial seizures. Only $15.2 \%$ patients had a family history of seizures and it indicates there is no significant association between the disease and inheritance. From the study finding $51.4 \%$ of the patient had a normal sleep pattern and 32.4 $\%$ with disturbed sleep and sleep pattern had no significant association with the disease.

The main aim of the treatment of epilepsy is to make the patient completely seizure free, or to reduce the frequency and severity of the seizure, if it cannot suppressed completely. The standard treatment of epilepsy is optimal use of anti epileptic 
drugs. In this study Levetiracetam (71.4) was the most commonly prescribed drug followed by the Phenytoin (31.4), Lorazepam (16.2), Clobazam and Sodium valproate (11.4). This finding was contrast with study conducted by Lim et al., 1997 in Singapore in which Carbamazepine was the most commonly prescribed drug followed by Sodium Valproate and Phenytoin. In another study conducted in Ethiopia by Rishe et al., 2015 Phenobarbitone was the most commonly prescribed drug.

Current study found that $59 \%$ patients were on polytherapy and $41 \%$ on mono-therapy these findings were differ from a study conducted by Rishe et al., 2015 where $78.6 \%$ patients received mono-therapy and from another study carried out by Lim et al., 1997 found that $62.7 \%$ patients were on mono-therapy. It is important to maintain patients on mono-therapy as compliance is better, side effects are less and there is no problem of drug-todrugs interactions and to yield better compliance. $52.4 \%$ of the patients received drugs orally and $26.7 \%$ received drugs both by oral and IV routes and only $21 \%$ by IV route. $84.9 \%$ of the patients were administered drug twice daily. Once daily dosing could be more appropriate to yield better patient compliance.

In the present study assessment of knowledge of patient's caregiver was carried out before and after counseling. It was found that most of the care givers were educated. $54.3 \%$ were graduates and $16.2 \%$ had a higher secondary level of education. Patient's care givers were close relatives such as partners, children, parents and siblings. Before the counseling session, most of the care giver $(80 \%)$ had a medium knowledge, $19 \%$ with low knowledge and only $1 \%$ with high knowledge. In the present study, it was able to identify that most of the patient's care givers lack the knowledge in terms of first aid measures during seizure. Most of them believed strongly in old age measures such as placing an object in the patient's mouth and holding keys or metal pieces in their hands during seizure is beneficial and appropriate to terminate an epileptic attack. In fact, placing an object in the patient's mouth can lead to tooth breakage or injure the hand of rescuer. Placing keys or metal pieces in the hands of the patients is also inappropriate and harms the patient or the rescuer. After the counseling session, $99 \%$ of the patient attained a higher knowledge and the remaining $1 \%$ had medium knowledge. It clearly indicates a significant association in the improvement of knowledge by the patient counseling. The provision of better care for people with epilepsy requires not only better knowledge and attitudes towards epilepsy, but also better information resources for care givers. Patient education was found to be an effective tool in improving health outcomes such as reducing the need for medication, reducing the duration of treatment and hospital stay, reducing the risk factors and improving the risk reducing behavior.

The patient's care giver must be clearly informed about the 'do's and 'don'ts during and after a seizure so that the patient is not harmed in the process of helping. The current first aid rules for a tonic-clonic seizure include removing all sharp objects from the vicinity of patient and protecting the head from injury. Place the patient in recovery position once the seizure stops. In case of frothing, tilt the head to one side so that the froth comes out of the mouth and prevents choking. Allow the patient to rest and don't feed the patient till consciousness is regained.

Quality of life is an important measure in epilepsy which is a chronic, debilitating condition and unique among the chronic illnesses due to its multi dimensional impact on psychosocial functioning. In the study the mean QOLIE score was $55.9 \pm 14.5$. The patients were found to have more distress associated with lower energy/fatigue with a score of $46.8 \pm 12.3$ followed by overall quality of life with a score of $48 \pm 17.3$ and emotional well being with a score of $53.4 \pm 16.9$. The patients were least distressed by the medication effects and social function with a score of $73.4 \pm 18.4$.

Assessment of quality of life relatively measures the outcome of treatment for epilepsy. The care of the epileptics must be taken in to account by considering the various factors influencing the quality of life in these patients and the management of epilepsy should be focused

\section{CONCLUSION}

The present study was conducted to evaluate the prescribing pattern of antiepileptic drugs and to determine the impact of patient counseling in improving the knowledge and quality of life in patients with epilepsy. The study was conducted in 105 in-patients diagnosed with epilepsy. This study was able to identify the prescribing trend in the tertiary care hospital. Levetiracetam was the most commonly prescribed drug in the study hospital. Regarding with the knowledge of the care givers of patients, most of them had a medium level of knowledge regardless of education. Female bystanders had a better knowledgeable about the disease. Counseling was found to be an effective tool in improving the knowledge of care givers. A considerable improvement in knowledge was observed after the provision of counseling which will surely reflects in patient care. Regarding with the quality of life of the patients, it was lower mainly in the affected areas like energy or fatigue and emotional well being. Seizure worry and social functioning are other domains which have some effects related to quality of life. Cognitive function and medication effects are of least importance. Through this study it was able to give proper counseling especially in areas where knowledge was lacking. This study may helpful to healthcare professionals in identifying the areas where the quality of life of the patient can be improved and also clearly defines the role of clinical pharmacist in providing information through patient counseling.

\section{Financial support and sponsorship: Nil.}

Conflict of Interests: There are no conflicts of interest.

\section{REFERENCES}

de Bittencourt PRM, Adamolekum B, Bharucha N, Carpio A, Cossio OH, Danesi MA, Dumas M, Meinardi H, Ordinario A, Senanayake 
N, Shakir R, Sotelo J. Epilepsy in the tropics: I. Epidemiology, socioeconomic risk factors and etiology. Epilepsia, 1996; 37 (11): 11211127.

Ekta Singh Dahiya, Man Mohan Mehndiratta, Krishna Kolappa Pillai. Plasma tetranectin as a potential clinical biomarker for epilepsy and correlation with clinical and social characteristics. International Journal of Epilepsy, 2017; 4 (1): 2-5.

(10): 1143-1151.

Jallon P. Epilepsy in developing countries. Epilepsia, 1997; 38

John Elliott, Bassel Shneker. Patient, caregiver, and health care practitioner knowledge of, beliefs about, and attitudes toward epilepsy. Epilepsy Behav, 2008; 12 (4): 547-556.

Lucretia Long, Andrew L. Reeves, Layne Moore J, Jessica Roach, Carolyn T. Pickering. An assessment of epilepsy patients' knowledge of their disorder. Epilepsia, 2000; 41 (6): 727-731.

Minumaria Shaju, Kollencheri Puthenveettil Vinayan, Suja Abraham. Knowledge, attitude and practice of parents regarding pediatric antiepileptic drug therapy. International Journal of Epilepsy, 2014; 1 (2): 57-63.

Nimabal SK, Venkatrao N, Basavaraj Pujar, Shalam, Shivakumar Ladde. Evaluation of anticonvulsant activity of alcoholic extract of Benincasa hispida (Thunb) Cogn. fruit extracts. Int. Res. J. Pharm., 2011; 2 (12): 166-168.

Shih-Hui Lim, Eng-King Tan, Christopher Chen. Pattern of anti-epileptic drug usage in a tertiary referral hospital in Singapore. Neurol J Southeast Asia, 1997; 2: 77-85.
Stacy Kuriakose, Emmanuel James, Anand Kumar. Assessment of knowledge of epilepsy in epileptic patients attending a tertiary care centre in Kerala, India. Int J Pharm Pharm Sci, 2014; 6 (7): 64-67.

Vanitha Rani N, Nisha Alice Varughese, Shankar V, Kannan G, Thennarasu P. Assessment of quality of life of patients with epilepsy in the neurology OPD of a tertiary care university hospital. Int. J. Res. Pharm. Sci., 2013; 4 (2): 141-145.

Wakjira Rishe, Muluneh Fromsa Seifu, Belayneh Kefale Gelaw, Thirumurgan Gunasekaran, Esayas Tadesse Gebremariam, Mustefa Ahmed Mohammed. Drug use evaluation of antiepileptic drugs in outpatient epilepsy clinic of bishoft general hospital, East Shoa, Ethiopia. Int. J. Res. Dev. Pharm. Life Sci., 2015; 4 (3): 1516-1528.

\section{How to cite this article:}

Lekshmi AA, Anjana A, Emmanuel SM, Sreelekshmi V, Selvin CDS. Evaluation of Prescribing Pattern of Antiepileptic Drugs and Assessment of Quality of Life of Epileptic Patients and the Knowledge of Their Care Givers. J App Pharm Sci, 2017; 7 (10): 152-156. 